\title{
...
}

NASA Technical Memorạndum 103664

$|N-3|$

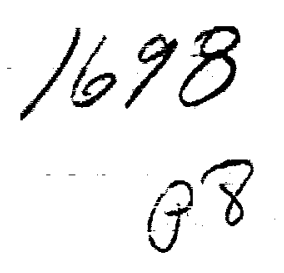

\section{Development of a Vibration Isolation Prototype System for Microgravity Space Experiments}

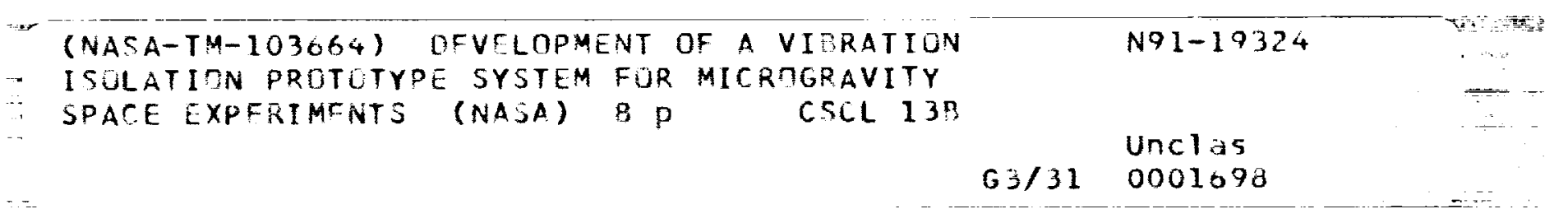

Kirk A. Logsdon, Carlos M. Grodsinsky, and Gerald V. Brown Lewis Research Center Cleveland, Ohio

Prepared for the

Twenty-Eighth Plenary Meeting of the Committee on Space Research cosponsored by COSPAR, ESA, and NASDA

The Hague, The Netherlands, June 25-July 6, 1990 
DEVELOPMENT OF A VIBRATION ISOLATION PROTOTYPE SYSTEM FOR MICROGRAVITY SPACE EXPERIMENTS

Kirk A. Logsdon," Carlos Y. Crodsinsky, * and Gerald V. Brown**

"Space Experiments Division, "Structures Division, Vational de conautics and Space Administration, Lewis Research Center. Cleveland, Ohio +4135

\section{ABSTRACT}

The presence of small levels of low-frequency accelerations on the space shuttle orbiters has degraded the "microgravity" environment for the science community. Growing concern about this microgravity environment has generated interest in systems that can isolate microgravity science experiments from vibrations. This interest has resulted primarily in studies of isolation systems with active methods of compensation.

This paper presents the development of a maznetically suspended, six-degree-of-freedom active vibration isolation prototype system capable of providing the needed compensation to the orbital environment. A design for the magnetic actuators is described, and the control law Eor the prototype system that gives a nonintrusive inertial isolation response to the system is also described. Relative and inertial sensors are used to provide an inertia! reference for isolating the payload.

\section{NOMEVCL.TTLRE} cross-sectional area of magnet pole face

ceq celative electromagnet damping coeffi-

$k_{p i}$ inertiai proportional gain consiant

celative electromagnet damping coeffi-

keq magnetic circuit isolator stitíness.

$\mathrm{Y} / \mathrm{n}$

cient, $Y$-sec/m

$F \quad$ force exerted by electromagnet, $y$

m payload mass, $\mathrm{kg}$

$g$ acceleration, $\mathrm{m} / \mathrm{s}^{2}$

go acceleration due to gravity, $\mathrm{m} / \mathrm{s}^{2}$

$\mathrm{V}$ number of ampere turns

$\mathrm{h}$ air gap between pole face and armature

$s$ Laplace variable

i current in the coil

t time, sec

$K_{d}$ derivative gain constant

$K_{d i}$ inertial derivative gain constant

$K_{i}$ integral gain constant

$K_{p} \quad$ proportional gain constant INTRODUCT ION

$u$ position of base

$\dot{u}$ velocity of base

ü acceleration of base

$x$ position of payload

$\mathrm{x}$ velocity of payload

$\mu_{0}$

permeability of free space

Interest in vibration isolation for microgravity experiments has increased within the microgravity science community as the flight program has progressed, and the small, but significant, levels of residual acceleration on the space shuttles (STS) have become more widely recognized and documented $/ 1,2,3 /$. These background accelerations are due to several sources characteristic of the orbiting carrier and the orbital environment. Very low frequency (dc to $10^{-3} \mathrm{~Hz}$ ) accelerations due to drag, tidal effects, and gravity gradients contribute sub-micro-g/go levels of acceleration to the environment. STS thruster activity can contribute $10^{-4}$ to $10^{-2} \mathrm{~g} / 80$ accelerations with significant duration, but these can be predicted and controlled. The most visible and troublesome contribution to most experiments is the moderate frequency $\left(10^{-3}\right.$ to $\left.10^{2} \mathrm{~Hz}\right)$ dynamic spectrum of accelerations with magnitudes in the range of $10^{-5}$ to $10^{-2} \mathrm{~g} / \mathrm{g}_{0}$. This dynamic background is due substantially to randow excitations from human activity on the orbiter. However, orbiter structure and flight systems also contribute observable intermittent and resonant accelerations to the background as the orbiter interacts with $i$ ts dynamic mechanical and thermal environment $/ 4 /$. 
The evolution of the Space Station Freedom design has led to many discussions of the potential limitations on long-term, low-gravity experimentation in this environment. It is now obvious that most of the true microgravity experiments will require "protection" from this randon, milli-g environment if valid and reproducible results are to be expected. Because a large part of the transient disturbances have a frequency range from $10^{-3} \mathrm{~Hz}$ to $1 \mathrm{~Hz}$, it is extremely difficult to design a passive isolation system with a resonance frequency at most $1 / \sqrt{2}$ times the lowest excitation frequency of interest, mainly the subhertz range.

The serious limitation of passive isolators is the absence of materials which have useful ranges of both low modulus (providing low frequency) and appropriate damping (to avoid large amplitude oscillation). Two-stage passive isolators can decrease the frequency. but limited damping leads to potentially unstable systems in the random excitation environment. A passive isolation system would not suffice because extremely low stiffness is necessary io isolate microgravity experiments with typical masses irom small disturbance frequencies. When there are direct disturbances on the payload. a low stiffness value is not desirable. Therefore, there is a tradeoff, and an optimal design would need to compensate for both direct disturbances, if present, and low-frequency base disturbances. Thus, active systems offer significant advantages over passive systems in the orbital acceleration environment because of their extremely small stiffnesses, which are needed to isolate against such low-frequency base disturbances, and their ability to adapt to direct disturbances for the optimal isolation of the payload, since the responses to these two excitations require conflicting solutions

\section{OVERVIEN}

An expriment payload allowed to "free float" inside the orbiter would have cotimal isolation, limited only by the magnitude of direct disturbances. However, the very low frequency accelerations due to drag, tidal effects, and gravity gradients would cause the payload to eventually drift into its boundary constraints (unless the orbiter were flown around the payload). It is apparent that some type of control would be necessary to keep the experiment payload within prescribed boundaries.

A vibration isolation system capable of producing a predictable, vell-characterized. and reproducible 'microgravity' environment must, by necessity, be actively controiled. in active control system to provide such environmental control xill be more complex than 7 passive system: however, the stiffness and damoing ratios achievable for a passive sysisti are not adequate for the needs of many "miciogravity" experiments.-

The optimal isolation of a payload. Iimited only by the volume constraints typical of a!: space-based payloads, will be produced by an inertially referenced isolation system. The feedforward/feedback closed-loop control configuration developed for the inertially referenced prototype system can provide the appropriate inertial contol to isolate a payload against the low-frequency disturbances that are encountered on the space shuttle orbiters and will be encountered on Space Station Freedom.

In electromagnetic actuator is the best nonmechanical means for actively controlling the payload. The force provided by such an actuator can be controlled with a feedback controller, and it is effective without contacting the payload, thus prohibiting any vibratory short-circuits to the surrounding support. Relative position feedback is necessary to stabilize an attractive actuator and to keep the payload within prescribed boundaries. In the presence of an umbilical, a negative relative stiffness can be introduced into the control law.

A noncontacting sensor is used to prevent external base disturbances from being transmitted through a physical connection. A closed-loop controller referenced to relative information would maintain the payload within its prescribed boundaries, and the ability of such a controller to reject disturbances would depend on the equivalent stiffness and damping of this relative-referenced system. The resulting physical response of such a system would simply be that of a spring-mass-damper system with variable parameters.

For the controller to optimally isolate the payload, the inertial information of the payload must be incorporated into the controller. By sensing the acceleration of the dynamic base. it can determine this information. This feedforward approach, coupled with the relative feedback discussed earlier, provides the necessary closed-loop system response to achieve a controlled "microgravity" envi ronment $/ 2 /$.

The controller for such an isolation systen must be capable of adjusting control parameters on the basis of the level and type of disturbance encountered. An analog controller would be more desirable due to fast response time: however, it would not be capable of tuning control parameters for varying types of disturbances. The versatility of a digitai controller provides this "adaptive" control capability. The use of a digital controllec also allows the control system engineer the $f$ reedom to reconfigure the controller through 
sof tware, rather than changing discrete hardware elements - thus reducing development time. When the prototype system progresses beyond the development stage, the controller configuration will likely contain both analog and digital elements. resulting in a hybrid controller. For such a system, a digital computer can monitor the system and send control commands to an analog subsystem where controller gains can be digitally programmed.

The prototype system presented in this paper employs these elements to realize inertial vibration isolation. The following sections describe these elements in more detail.

\section{Actuators}

There are two types of electromagnetic actuators: a Lorentz force actuator and an attractive electromagnetic actuator. The first has a linear current-to-force relationship and the second has a nonlinear relationship between the coil current and the force. and an inversely nonlinear relationship between the air gap between the pole face and armature and the force. The attractive actuators were used in this six degree-of-freedom (DOF) prototype system because the force-to-input power capability is much greater. In the laboratory, one must offset the constant loading of a ig field, and attractive actuators provide the additional force with a minimal increase in input power.

Because these actuators produce force in only one direction. a pair of actuators nusi be used in both the horizontal and vertical directions (at each point) to attain bidirectiona: actuation. The relationship for the force exerted by an attractive electromagnet is

$$
F=\frac{\mu_{0} d N^{2} i^{2}}{h^{2}}
$$

As can be seen by equation (1), the force is related nonlinearly to the coil current and inversely nonlinearly to the air gap. As more current is supplied to the actuator by the controller, the altractive force increases. thus drawing the armature closer to the magnet This causes the air gap to decrease, which also draws the armature closer to the magne: This interaction illustrates the open-loop instability of this actuator. The actuator however, can be made stable by closing a control loop around the position of the armatirs relative to the actuator to achieve a positive equivalent stiffness. This approach :0 stabilizing such a system is described in many papers $: 5.6 \%$

By choosing the appropriate cross-sectional area of the actùator pole face and the number oi ampere turns in the coil, the actuator can be designed to $f i t$ any volume and powe? constraints associated with the payload design. The actuators designed for this prototype system have two coils, wired in parallel, with approximately 1500 turns each and a core cross-sectional area of $6.45 \mathrm{~cm}^{2}(1 \mathrm{in.}-)$. With a current of 21 and an air gap of $0.22 \mathrm{~cm}$ $(0.125$ in.), these actuators can support a weight of approximately 667 y ( 150 lb) each. This high force capability will allow the platform to be controlled throughout the entire trajectory flown by the VASA Lewis Learjet during microgravity flights, where forces can reach 2 to $2.5 \mathrm{~g}$ during pull-up maneuvers. Actuators for space flight payloads wi!! be designed with much smaller forces and, thus, will be significantly smaller in size.

\section{Sensors}

Noncontacting relative position sensors are required to maintain the experiment payload within its work volume. The position error detected by the sensor as the payluad moves with its volumetric boundaries is used by the controller to return the payload to its nominal position relative to the base and to prevent collisions with the walls. These sensors must be noncontacting to prevent any base disturbances from being transmitted to the payload through the sensor itself.

The relative position sensors currently being used on this prototype system are eddy-current probes. Their linear operating range is a function of the radius of the probe head. These probes provide an operating range of approximately $1.27 \mathrm{~cm}(0.5 \mathrm{in.})$. Therefore. the movement of the payload in the work volume is limited to approximately $\pm 0.635 \mathrm{~cm}(=0.25 \mathrm{in}$.) . These sensors give an output signal of $7.87 \mathrm{~V} / \mathrm{cm}(20 \mathrm{~V} / \mathrm{in}$. $)$. This signal is conditioned to provide a corresponding range of -10 to $10 \mathrm{~V}$ to the controller.

As wentioned earlier, an inertial sensor is required to optimally isolate the experimental payload from the dynamic base environment. Inertial information about the dynamic base environment coupled with the relative information would provide the necessary data for inertial isolation. An accelerometer mounted to the base of the system provides the required inertial reference.

The accelerometers used in this prototype system provide an output signal of $1.33 \mathrm{mt} / \mathrm{g}$, where $g \equiv 9.81 \mathrm{~m} / \mathrm{s}^{2}\left(32.2 \mathrm{ft} / \mathrm{s}^{2}\right)$. This signal is ac coupled to eliminate any dc of ${ }^{2}$ sets (i.e., constant loading due to gravity) since the experiment can only be isolated from 
time-varying disturbances. (Variations due to temperature fluctuations are too slow to significantly affect the output.) The signal is then amplified to provide an operating range of -5 to $5 \mathrm{~V}$, corresponding to -0.5 to $0.5 \mathrm{~g}$, to the controller.

\section{Cont roller}

The control algorithm for the prototype system was implemented on a desktop personal computer. An analog-to-digital $(\mathrm{A} / \mathrm{D})$ converter with 16 input channels was used to read the sensor signals. The $A / D$ converter has 12 -bit resolution and is configured for $\pm 10-V$ inputs. The output signals generated by the controller are sent to a digital-to-analog $(D / A)$ converter. The $D / A$ converter has six output channels. also with 12-bit resolution. Each channel is configured for $\pm 10-V$ output. These outputs control the power supplies that drive the electromagnetic actuators.

There are 12 input signals to the controller: 6 relative position signals and ij acceleration signals. These signals are resolved into 5 components to determine the inertial motion of the payload in all six DoF.

Vine output signals control the electromagnetic actuators. Vormally. $t$ actuators at each control point (12 in total) would be required to provide bidirectional force capability on the payload in all DOF; however, gravity is used as the restoring force in ihe rertira: direction on the prototype system.

The controller is derived from the equation of notion of a one-Dof model bor the iner:ia: isolation system. In reference to Figure 1 , the equation of notion is as follows:

$$
m \frac{d^{2} x}{d t^{2}}+c_{e q}\left(\frac{d x}{d t}-\frac{d u}{d t}\right)+c_{e q} \frac{d u}{d t}+k_{e q}(x-u)+k_{e q} u=0
$$

The relative terms in equation (2) are measured with respect to the base of the system. The relative velocity is determined by digitally differentiating the position signal in the computer, and the inertial terms are determined by digitally integrating the acceleration or the base of the system. As one can see. by combining the terms vith appropritite gains. the payload can be inertially referenced.

The vertical and horizontal motion at each control poit on the prototype sysian are controlled independently. The type of controller uset is a variation of a typica! proportional + integral + derivative (PID) controlier. The relative posi:ion of the experiment payload is the feedback signal used io maintain the payload in its work volume. The acceleration of the base is the feedforward signal used in conjunction with the relative position signal to inertially reference the payload. The following is the control law used to inertially isolate the payload. The control block diagran for one DOF is shown in Figure 2.

$$
i=k_{p}\left[(x-u)+k_{p i} u\right]+k_{d}\left[(x-\dot{x})+k_{d i} \dot{u}\right]+k_{i j} j(x-u) d t
$$

The control curcent is proportional to the displacement of the payload from the desired position (i.e., stiffness). the velocity of the payload (i.e., damping), and the accumulated error between the desired position and the actual position. The last term will maintain the payload in it 5 work volume.

The gain constants are determined to give the best overall response of the system to its base disturbances. By setting the gains appropriately. the controller can give the inertial stiffness and damping response desired.

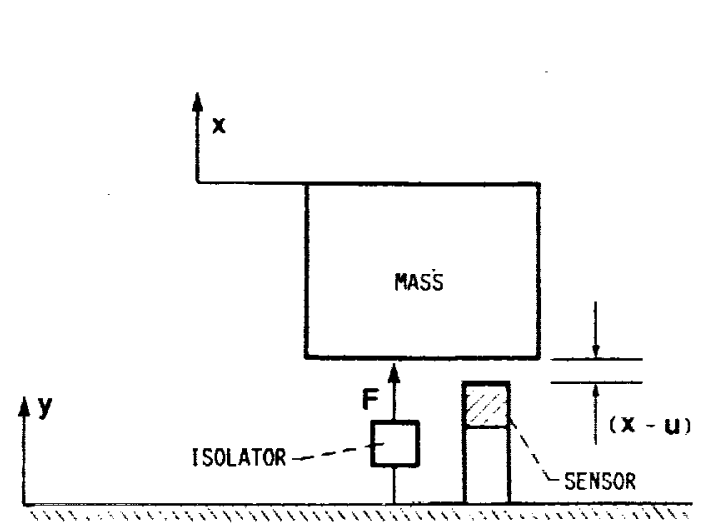

FIG. i. OME-DEGREE-OF-FREEDOM ACTIVE ISOLATION SYSTEM.

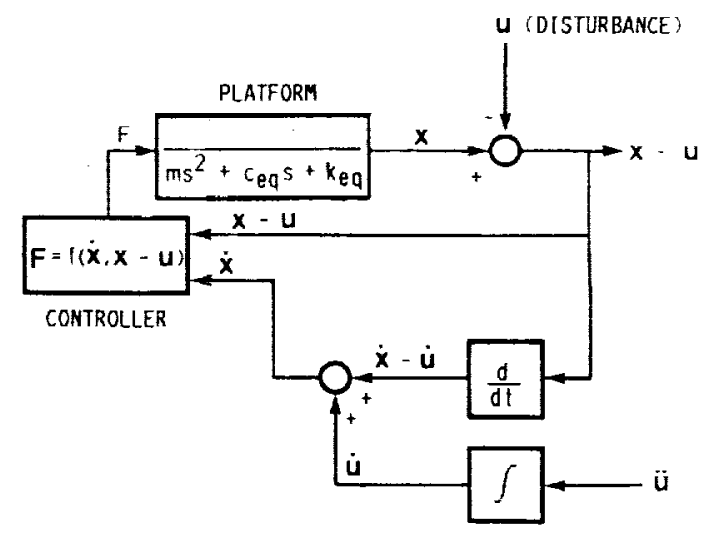

FIG. 2. ONE-DEGREE-OF-FREEDOM INERTIAL CONTROL SYSIEM. 


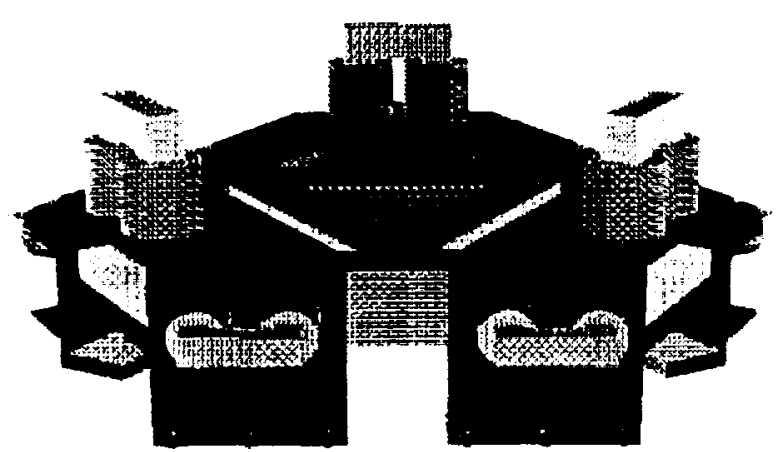

(a) PERSPECTIVE VIEW.

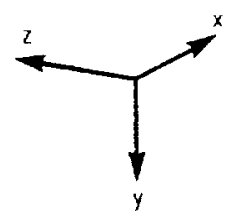

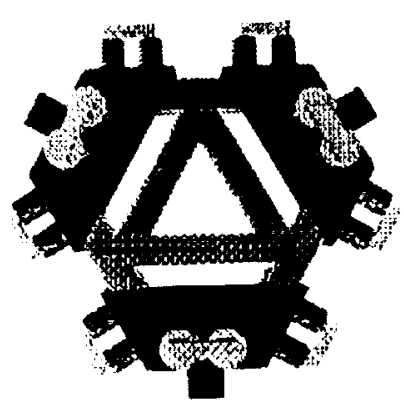

(b) IOP VIEW.

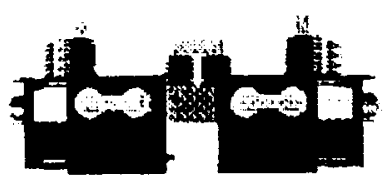

(c) SIDE VIEW

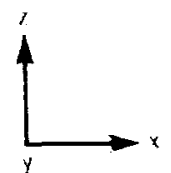

FIG. 3. SIX-DEGREE-OF-FREEDOM INERIIAL ISOLATION SYSTEM IAYOUT.

Hardware

The prototype system described in this paper is designed for six-Dof vibration isolation control. The inertially controlled platform is a hexagon actuated at three equally spaced points from its center of gravity. Figure 3 shows the physical layout of the sysiem where the hexagonal platform is shown internal to the actuator pod locations.

Two relative and inertial sensors are located at each control point giving the relative and inertial parameters of the payload in the horizontal and vertical planes. Three attractive electromagnets are located at each point so that the payload is actuated in two dimensions per location. Thus, by combining the actuation of these points, we can control six DOF.

The prototype system hardware consists of an isolation platform and a base mount for the electromagnetic actuators. The actuators are mounted on housings that extend over the platform at each of the three control points - the platform functions as the armature to complete the magnetic circuit. The controller operates on each point independently.

At present the system is configured for only three-Dof vertical translation, and rotations about the horizontal axes are the controlled DOF. The translational motion in the horizontal plane is constrained by a gimble located at the center of gravity of the controlled platform. The rotation about the vertical axis is free to move. Full six-DOF control will be completed as the necessary hardware becomes available.

RESULTS

The entire configuration is mounted on a pneumatically controlled optical isolation table. The pneumatic valves on the table are adjusted to force the table to oscillate when disturbed. This simulates a periodic jase disturbance for testing purposes. The table oscillates at a frequency of approximately $0.6 \mathrm{~Hz}$.

In order to demonstrate the inertial isolation capabilities under these conditions, a low-power laser is mounted to the isolation platform. The laser beam is reflected of a mirror located approximately $100 \mathrm{ft}$ from the test $r i g$ and displayed on a nearby wall. This "moment arm" dramatically exaggerates the movement of the laser as it oscillates. Cnce the isolation platform is lifted to offload gravity, the inertial isolation control can be switched on and of at the computer's keyboard to demonstrate the system is isolation capability.

When the inertial controller is switched off, the laser beam follows the movement of the base as it oscillates. When switched on, the beam holds steady on the wall - since it is inertially referenced. The unique aspect of this technology is the nonintrusive inertial feedforward/feedback compensation. 


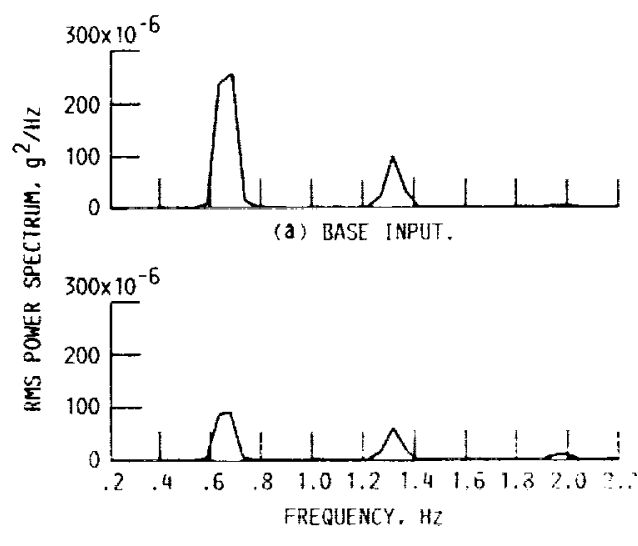

(b) ISOLATED PAYLOAO RESPONSH.

FIG. 4. POWER SPECIRUM OF ACCELERATION.

Preliminary measurements show that the three-Dof isolation system contijuration ban attenuate this disturbance by about 63 percent. In providing an fxcitation Eor tae inertially referenced platform. the periodic disturbance was a complicated six-DOF notian where the prototype was configured for three-Dof control; thus, the energy associated w: the noncontrolled directions coupled into the platform through the gimbal. Howeve. ine power spectrum shown in Figure $t$ illustrates the systems ability in attenuating such 1 complicated low-frequency disturbance by inertially referencing the payload. Once the remaining six-DOF hardware is completed. the coupling through the gimbal constraint will be eliminated, and the payload acceleration will be limited solely by the volume constraint or the system in reducing transmitted disturbances.

\section{CONCLLDING REMARKS}

In conclusion, it is apparent that actiog isolation systems will have to be used in the dynamic environment of manned orbiters if reproducible "microgravite exorimeniat:or. results are to be expected. The description for the design and contro! uf a roninrrus:ta. inertially isolated six-degree-of-freedon (DOF) sustem vas presented, and prefininai: results were given of its effectiveness in solving the low-frequency "yicrogras: isolation problem. These preliminary results were obtained to demonstrate the isolation capability of inertially referencing a payload. Once the six-Dof system is iully operational, the coupling effects due to the gimbal constraint will be eliminated. The use of an air table or pendulous mass would have afforded one the luxury of eliminating tine energy coupling into the system; however, this would not have demonstrated the :ul! capability of such a system in response to a realistic multidimensional disturbance. The completion of the remaining DOF is scheduled for test in the autumn of 1090. and a $i u l l y$ active system will be flown on low-gravity trajectory flights, for demonstration, in $199 !$.

\section{REFERENCES}

1. C.M. Grodsinsky, and G.V. Brown, Low Frequency Vibration Isolation Techno!ogy for Microgravity Space Experiments. NASA TY-1014t8, Vational deronautics and Spact: Administration, Lewis Research Center, Cleveland, OH (Sept. 1989).

2. C.M. Grodsinsky, and G.V. Brown, Nonintrusive Inertial Vibration Isolation Technoiogy for Microgravity Space Experiments, NASA TM-102386. Vational teronautics and Space Administration, Lewis Research Center, Cleveland, OH (Jan, 1990).

3. H. Hamacher. Simulation of Heightlessness, Materials Sciences in Space, B. Feuerbacher. H. Hamacher, and R.j. Naumann, eds., Springer-Verlag, Vew York, 1986, pp. 31-51.

4. C.R. Baugher, ed., Measurement and Characterization of the Acceleration En:ironment or: Board the Space Station: Proceedings of a Conference Implemented by Teledyne Brown Engineering, Guntersville, AL, tug. 11-14.1986, NASA, Washington, $\bar{D} \bar{C}, 1990$.

5. C.M. Grodsinsky, Development and Approach to Low-Frequency Microgravity Isolation Systems, NASA TP-2984, National deronautics and Space Administration, Levis Research Center, Cleveland, OH (dug. 1990).

6. R.R. Humphris, R.D. Kelm. D.X. Lewis, and P.E. Allaire, Effect of Control Algorithms an Magnetic Journal Bearing Properties. L. Eng. Gas Turbines Power. 108. $\neq 4$ pp. 634.6.2? $(1986)$. 


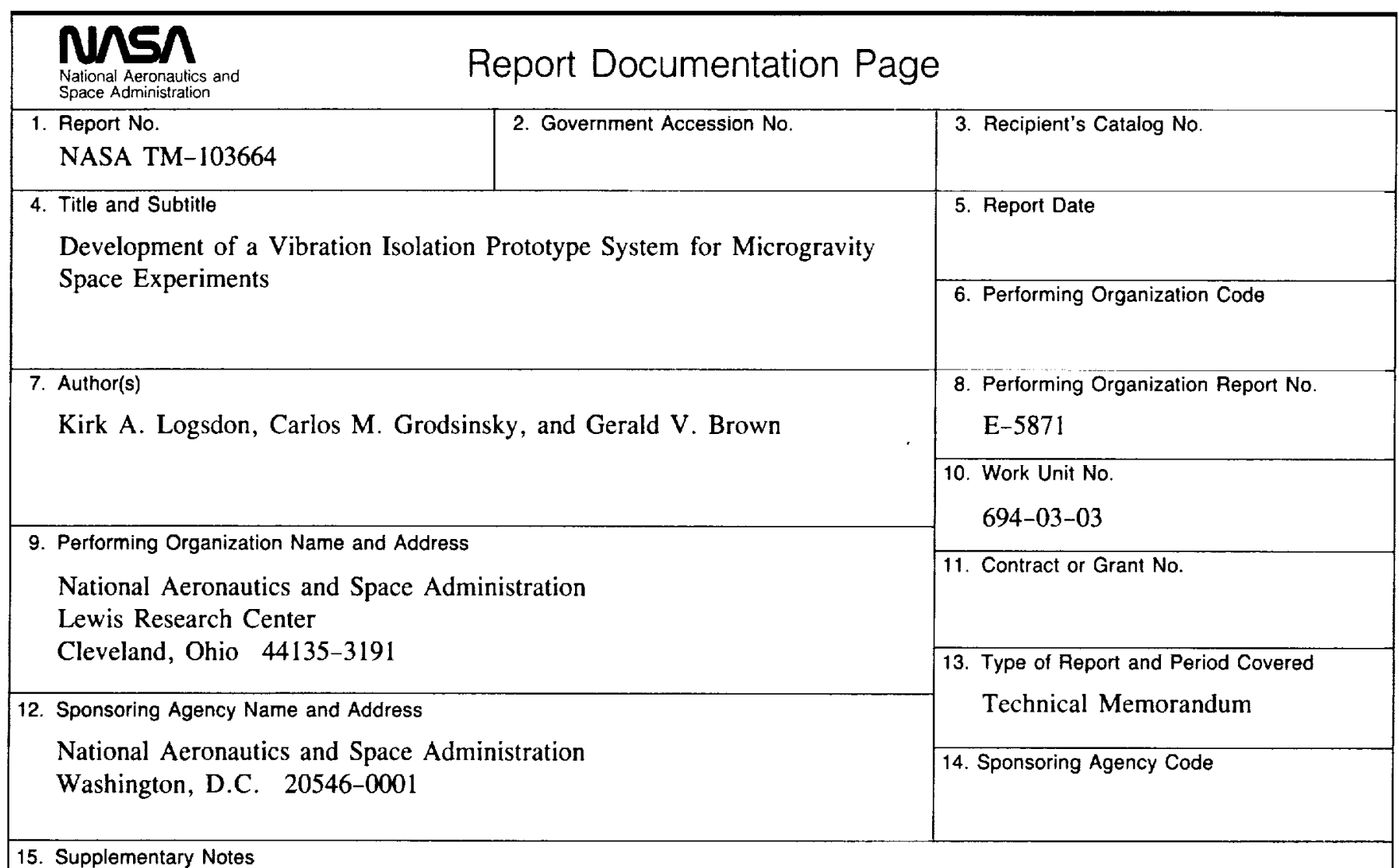

Prepared for the Twenty-Eighth Plenary Meeting of the Committee on Space Research cosponsored by COSPAR, ESA, and NASDA, The Hague, The Netherlands, June 25-July 6, 1990.

16. Abstract

The presence of small levels of low-frequency accelerations on the space shuttle orbiters has degraded the "microgravity" environment for the science community. Growing concern about this microgravity environment has generated interest in systems that can isolate microgravity science experiments from vibrations. This interest has resulted primarily in studies of isolation systems with active methods of compensation. This paper presents the development of a magnetically suspended, six-degree-of-freedom active vibration isolation prototype system capable of providing the needed compensation to the orbital environment. A design for the magnetic actuators is described, and the control law for the prototype system that gives a nonintrusive inertial isolation response to the system is also described. Relative and inertial sensors are used to provide an inertial reference for isolating the payload.

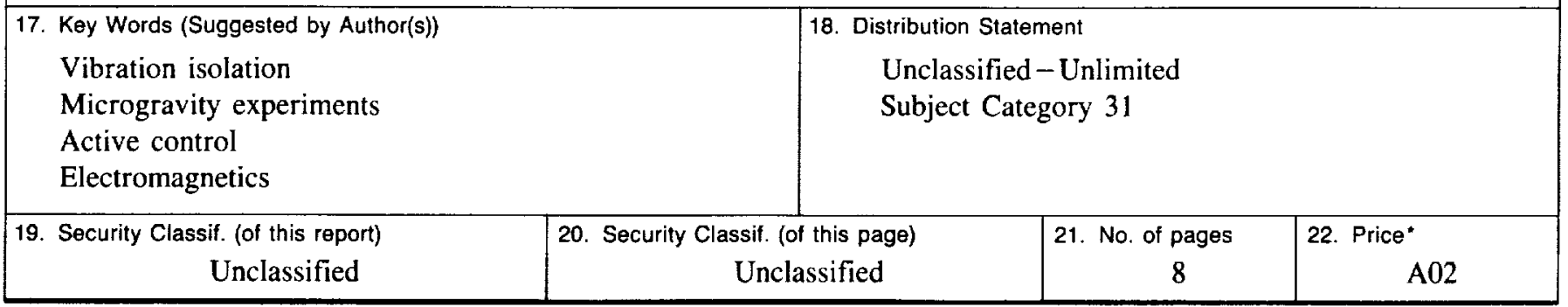

NASA FORM 1626 OCT $86 \quad$ "For sale by the National Technical Information Service, Springfield, Virginia 22161 
. 